MATEC Web of Conferences 11,01028 (2014)

DOI: $10.1051 /$ matecconf / 20141101028

(C) Owned by the authors, published by EDP Sciences, 2014

\title{
Effet du flambage sur les pipes en composite stratifiés munis d'entailles
}

\author{
Halima Chenine $^{1}$, Djamel Ouinas ${ }^{1}$, Saadi Mohamed ${ }^{1}$, Beladam Djamal ${ }^{1}$ \\ ${ }^{1}$ Laboratoire de modélisation numérique et expérimentale des phénomènes mécaniques \\ Département de Génie Mécanique-Université Abdelhamid Ibn Badis de Mostaganem-Algérie
}

\begin{abstract}
Résumé. Dans l'industrie (aéronautiques, automobiles, ...) les composites stratifiés se sont imposés pour la fabrication des pièces de structures primaires, grâce à leurs performances, leur qualité, légèreté et souplesse de forme. Cependant l'utilisation de ces matériaux est assujettie à la connaissance de leur comportement mécanique en statique comme en dynamique et également à la possibilité de le simuler. En effet, les matériaux composites ont un comportement particulier, et ceci est du essentiellement à deux facteurs. Le premier facteur résulte du comportement anisotrope du matériau utilisé dans la couche. Le deuxième facteur dérive de la stratification qui consiste à l'empilement de plusieurs couches, ce qui favorise l'effet de cisaillement transversal. Les structures minces en composites qui sont abondamment utilisées, deviennent instables lorsqu'elles sont sollicitées à des chargements de nature mécanique ou thermique conduisant au flambement. La principale cause de ce phénomène complexe, est le caractère aléatoire des imperfections initiales (en termes de géométrie, de matériau, ou de conditions aux limites) de la structure. Dans ce papier, l'analyse du flambage a été effectuée sur des pipes en composite stratifiés avec et sans entaille carrée, en ayant recours a la simulation numérique a l'aide de la MEF. Le pipe stratifié comporte plusieurs couches alternées de la manière suivante $(\Theta /-\Theta)_{5}$. La résistance au flambage des pipes est mise en évidence en fonction de l'orientation des fibres. Les résultats montrent que la charge de flambage est plus grande pour les plus petits diamètres, et surtout pour les orientations $\left(20^{\circ} \%-20^{\circ}\right)$, ce qui augmente le risque de la rupture. Et pour la distribution des contraintes de Von mises, la concentration la plus importante est pour les orientations $\left(20^{\circ} /-20^{\circ}\right)$ et les plus faibles au niveau de l'orientation $90^{\circ}$. Donc l'ampleur des contraintes dépend fortement de l'orientation des fibres de chaque pli. Mots clés -Entaille carrée, Concentration de contraintes, Flambage, Pipe en composite, Analyse par éléments finis.
\end{abstract}

\section{Introduction Générale}

Le moyen le plus fiable pour le transport des fluides est le pipe. Selon la nature du produit à transporter, le pipe est fabriqué en aluminium, acier, pvc ou en composite.

$\mathrm{Vu}$ les nombreuses défaillances remarquées lors de l'exploitation des pipes en aciers, exposées aux milieux corrosifs, la plupart des recherches [1] se sont penchées actuellement vers la construction de nouveaux pipes performants en matériaux composites. Pour permettre une meilleure construction, il est nécessaire de faire un bon dimensionnement des différents paramètres géométriques ou mécaniques pour assurer un rapport adéquat entre le poids et la résistance. C'est dans ce contexte que plusieurs travaux sont lancés dans le domaine de la conception et la modélisation des pipes en composite en vue de prolonger leurs durée de vie et de rationaliser leurs exploitations. Dans ce cadre, nous pouvons citer les travaux de Beakou et al [2] qui ont effectué une analyse fiabiliste sur un pipeline en composite avec un angle d'orientation de fibres $( \pm \theta)$ [3].

Les structures minces en composites, deviennent instables lorsqu'elles sont sollicitées à des chargements de nature mécanique ou thermique, conduisant au flambement qui est devenu une inquiétude majeure [4-5].

Les entailles sont aussi les zones de naissance des microfissures qui se propagent jusqu'à la ruine de la structure.
Afin de prédire l'apparition du phénomène d'endommagement, une étude des relations pouvant exister entre les défauts géométriques et l'orientation des fibres, du pipe stratifiée, ainsi que les propriétés mécaniques du matériau pour résister à la naissance de fissures, menant à la dégradation totale de notre tube.

\section{Modèles et maillage du pipe stratifiée sans entailles}

Pour notre application, nous avons choisi un pipe en composite stratifié de diamètre $\mathrm{d}=800 \mathrm{~mm}$ et d'une stratification de 10 couches alternées, la séquence d'empilement est $\left[\Theta^{\circ} /-\Theta^{\circ}\right]_{5}$, chacune a une épaisseur de $0.176 \mathrm{~mm}$. Le pipeline possède une longueur totale de $3000 \mathrm{~mm}$. Le matériau choisi est en carbone/Epoxy, T700/E avec des caractéristiques mécaniques données au tableau 1.

Tableau 1. Propriétés mécaniques du matériau du pipe $(\mathrm{T} 700 / \mathrm{E})$

\begin{tabular}{|c|c|}
\hline Propriétés & $(\mathbf{T 7 0 0} / \mathbf{E})$ \\
\hline $\mathrm{E}_{1}(\mathrm{MPa})$ & 143120 \\
\hline $\mathrm{E}_{2}(\mathrm{MPa})$ & 6672 \\
\hline$v_{12}$ & 0.26 \\
\hline
\end{tabular}




\begin{tabular}{|c|c|}
\hline $\mathrm{G}_{12}(\mathrm{MPa})$ & 3390 \\
\hline $\mathrm{G}_{13}(\mathrm{MPa})$ & 3390 \\
\hline $\mathrm{G}_{23}(\mathrm{MPa})$ & 1914 \\
\hline
\end{tabular}

Pour déterminer la fiabilité de la conception de cette structure vis à vis du flambage, la variabilité de plusieurs paramètres doit être prise en compte. Les pipes sont encastrés à l'une des extrémités, l'autre est soumise à un déplacement imposé. Notre structure a été modélisée par 9625 éléments quadrilatéraux de type S8R totalisant 33846 degrés de libertés. Trois points d'intégration de Gauss par pli sont considérés.
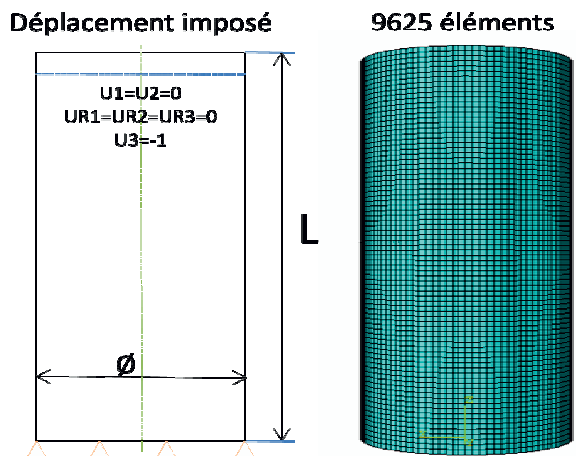

$/ 7 / / 7 / 7 / 7 / \quad / 7 / 7$

$\mathrm{U} 1=\mathrm{u} 2=\mathrm{u} 3=\mathrm{uR} 1=\mathrm{uR} 2=\mathrm{uR} 3=0$

Fig. 1. Representation des conditions aux limites et maillage du pipeline sans entaille.

\section{Evolution du paramètre de flambement pour les pipes sans entailles}

Dans cette partie de l'étude le but est de voir l'évolution du coefficient de flambement, en fonction de l'orientation des fibres sous l'effet d'un déplacement imposé U3, pour un pipe parfais sans défauts géométriques. Les résultats obtenus ont été traduits par les courbes ci-dessous.

\subsection{Variation du diamètre du pipe}

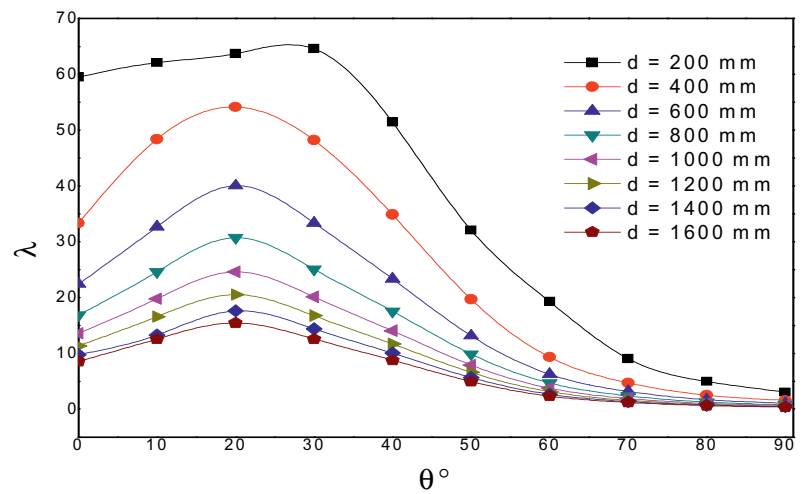

Fig. 2. Influence de la variation du diamètre du pipe sur le paramètre de flambement en fonction de $\theta$
La figure 2 montre l'évolution du coefficient de flambement en fonction de l'orientation des fibres du matériau composite, pour différents diamètres du pipe et pour un déplacement imposé de $\mathrm{u} 3=-1 \mathrm{~mm}$. On remarque que le coefficient de flambement atteint des valeurs maximales importantes, lorsque les fibres sont orientées dans un intervalle variant de $10^{\circ}$ à $30^{\circ}$. Les valeurs minimales sont obtenues lorsque l'orientation des fibres est comprise entre $60^{\circ}$ et $90^{\circ}$.

\subsection{Variation des épaisseurs du pipe}

La figure 3 montre la variation du coefficient de flambement en fonction de l'orientation des fibres pour différentes valeurs de l'épaisseur du pipe. La représentation est faite pour quatre épaisseurs différentes, $\mathrm{e}=2 \mathrm{~mm}, 4 \mathrm{~mm}, 6 \mathrm{~mm}$ et $8 \mathrm{~mm}$ pour un diamètre de 800 $\mathrm{mm}$.

On constate que le paramètre de flambement est maximal pour l'orientation $20^{\circ}$ des fibres. Par ailleurs, lorsque l'angle $\theta$ est supérieur à $70^{\circ}$, les valeurs de la charge critique sont quasiment constantes.

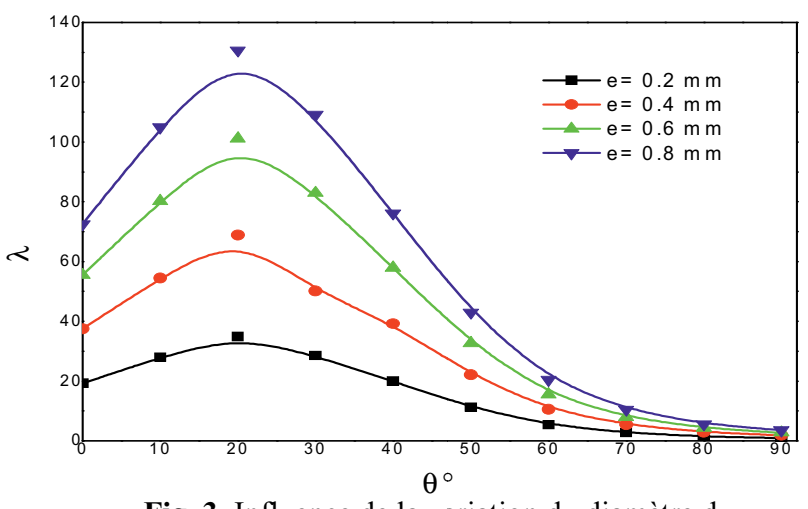

Fig. 3. Influence de la variation du diamètre du pipe sur le coefficient de flambement en fonction de $\theta$

\subsection{Variation du déplacement imposé du pipe}

La figure 4 montre que le paramètre $\lambda$ est maximal entre l'inclinaison de fibres variant de $10^{\circ}$ et $20^{\circ}$ par contre les valeurs minimales sont à partir de $\theta^{\circ}>50^{\circ}$.

On constate que $\lambda$ maximale est pour le plus petit déplacement imposé.

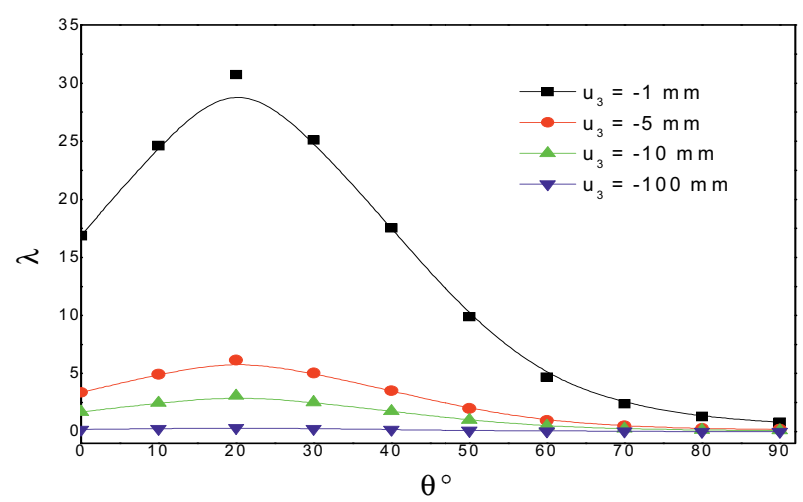

Fig. 4. Influence de la variation du déplacement imposé appliqué, sur le paramètre du flambement en fonction de $\theta$ 


\section{Evolution du paramètre de flambement pour les pipes avec entailles}

\subsection{Charge critique de flambement pour un pipe munie d'une seule entaille}

Dans cette partie d'étude on considère un pipe en carbone/époxyde de longueur $\mathrm{L}=3000 \mathrm{~mm}$ et de diamètre $\mathrm{d}=800 \mathrm{~mm}$, L'épaisseur de chaque plis du pipe a été fixée à e $=0.176 \mathrm{~mm}$.

Des défauts géométriques sont prévus, sous forme d'une seule, deux, puis trois entailles de forme carrées. Les pipes sont encastrés à l'une des extrémités, l'autre est soumise à un déplacement imposé $\mathrm{u}_{3}=-1 \mathrm{~mm}$.

Dans le calcul nous avons employé la méthode des éléments finis. Nous avons utilisé des éléments quadrilatéraux avec un maillage raffiné et structuré au voisinage de l'entaille comme le montre la figure 5.

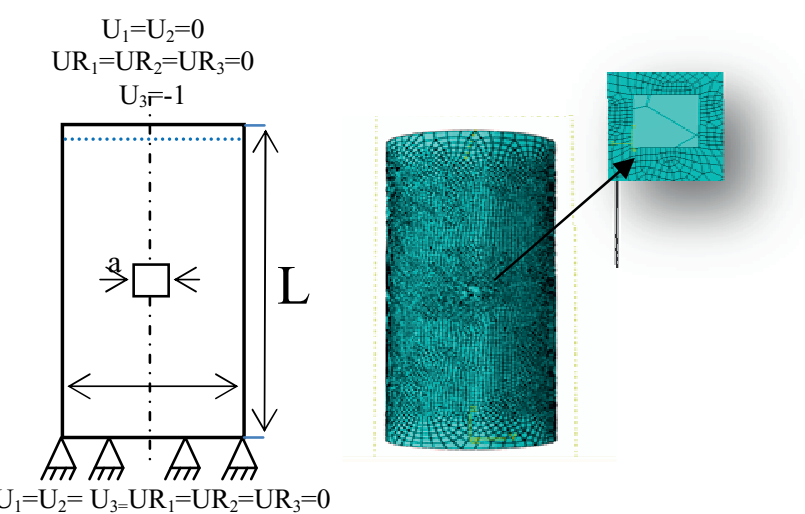

Fig. 5. Représentation des conditions aux limites

et maillage du pipeline muni d'une entaille carré

La figure 6. Illustre la variation du coefficient de flambement en fonction de l'orientation des fibres pour différentes entailles. le paramètre $\lambda$ est maximum quand l'angle d'inclinaison des fibres est orienté vers $20^{\circ}$ dans la majorité des cas étudiés, puisque c'est une entaille carrée, on a changé la taille du coté on a pris a $=6 \mathrm{~mm}$, $10,16,20$ et $40 \mathrm{~mm}$, comme le montre la figure. On note aussi que plus la taille du défaut est grande plus la structure est faible.

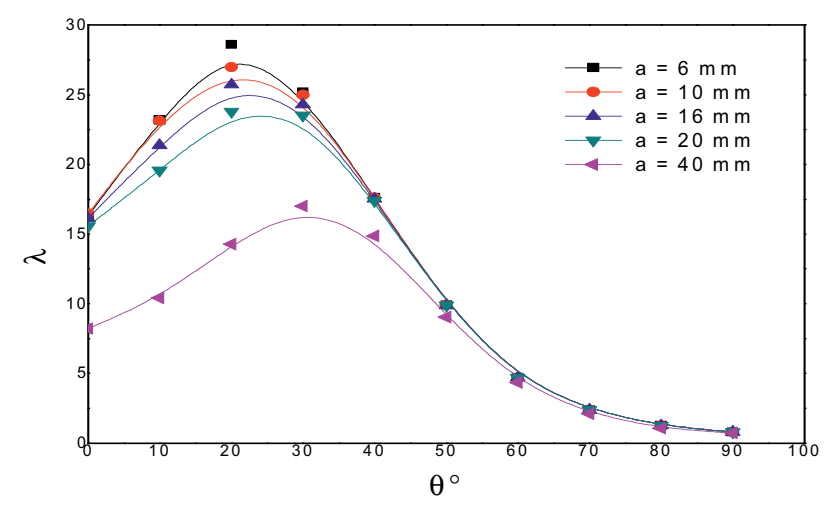

Fig. 6. Influence de la taille de l'entaille sur le facteur de flambement en fonction de $\theta^{\circ}$, pour un pipe muni d'une seule entaille.

\subsection{Charge critique de flambement pour un pipe munie de deux entailles}

\subsubsection{Cas de deux entailles: une située milieu du pipe l'autre coté encastrement}

La partie suivante consiste à mettre deux entailles l'une au milieu du pipe, l'autre avec les mêmes dimensions, située à $180^{\circ}$ par rapport à la première et juste à coté de l'encastrement, comme le montre la (figure 7). Les résultats obtenus sont illustrés par la (figure 8). la présentation a été faite pour deux dimensions d'entailles $\mathrm{a}=10 \mathrm{~mm}$ et $\mathrm{a}=16 \mathrm{~mm}$. Le coefficient de flambement est plus important dans l'intervalle $10^{\circ}-30^{\circ}$ et atteint une valeur critique $\lambda=28.425$ dans $1^{\prime}$ 'orientation des fibres de $20^{\circ}$ et cela pour l'entaille de $10 \mathrm{~mm}$ de coté.
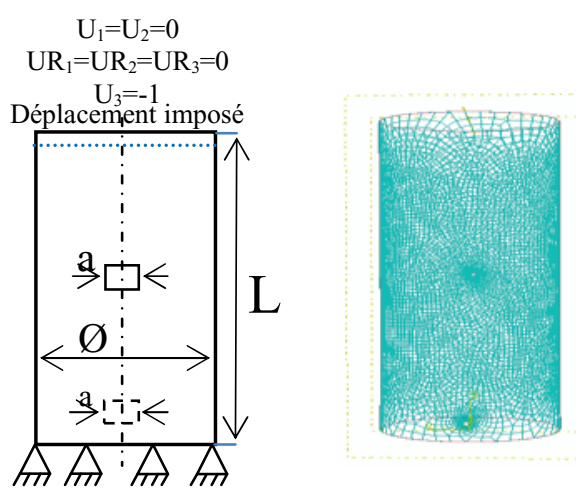

Fig. 7. Représentation des conditions aux limites et maillage du pipeline muni de deux entaille carré ( une situé coté encastrement).

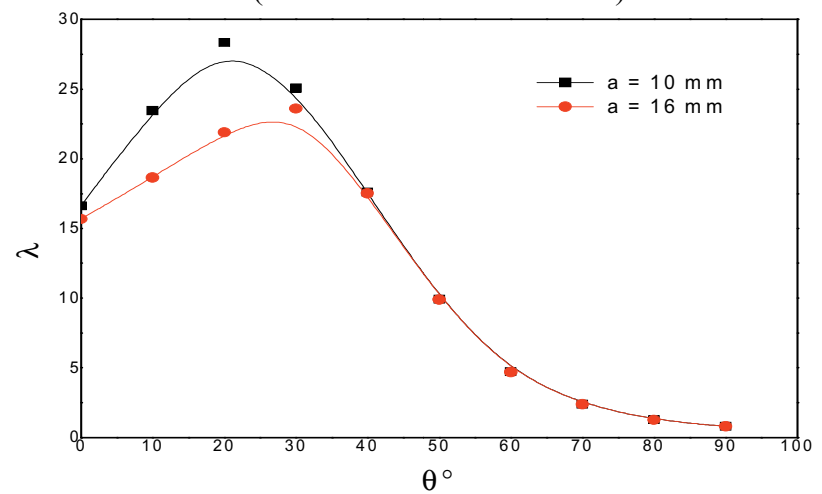

Fig. 8. Influence de la taille de l'entaille sur le facteur de flambement en fonction de $\theta^{\circ}$, pour un pipe à deux entailles. ( Entaille située du coté de l'encastrement).

4.2.2 Cas de deux entailles: une située milieu du pipe l'autre coté déplacement imposé

La même procédure a été faite mais la deuxième entaille se situe du coté du déplacement imposé (figure 9). Les résultats ont été groupés dans la (figure 10). On remarque que l'allure des deux courbes est semblable et le coefficient de flambage atteint une valeur maximale $\lambda=27.173$. 


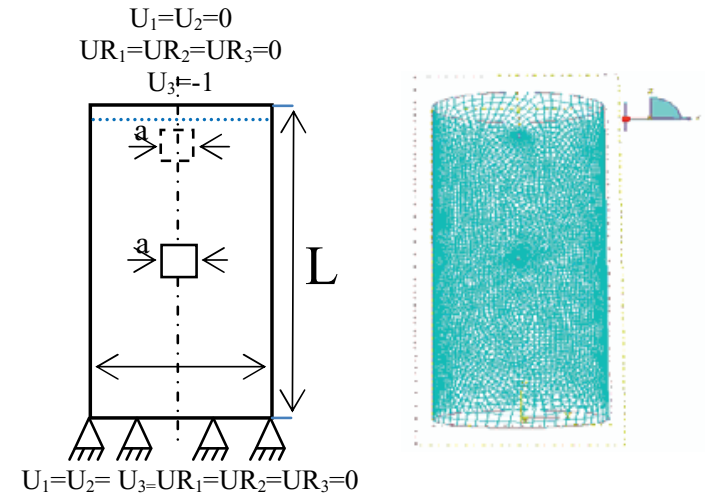

Fig. 9. Représentation des conditions aux limites et maillage du pipeline muni de deux entaille carré( une située coté déplacement).

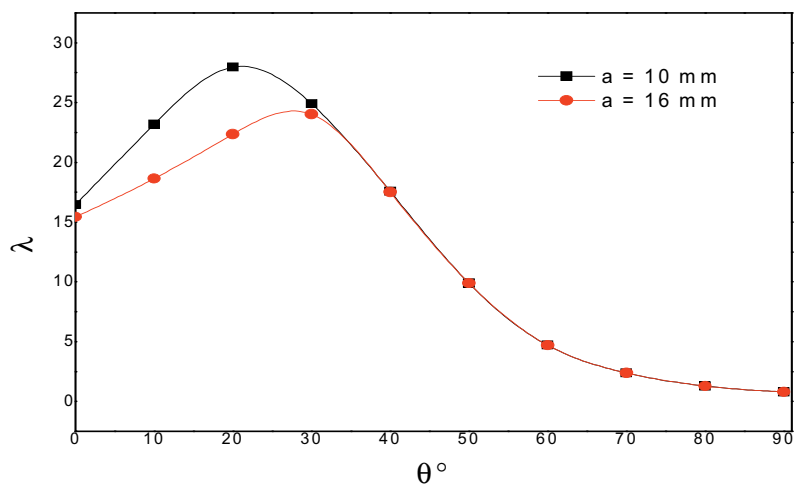

Fig. 10. Influence de la taille de l'entaille sur le facteur de flambement en fonction de $\theta^{\circ}$, pour un pipe à deux entailles. ( Entaille située du coté déplacement imposé).

\subsubsection{Cas de deux entailles: une située milieu du pipe l'autre en face coté opposé}

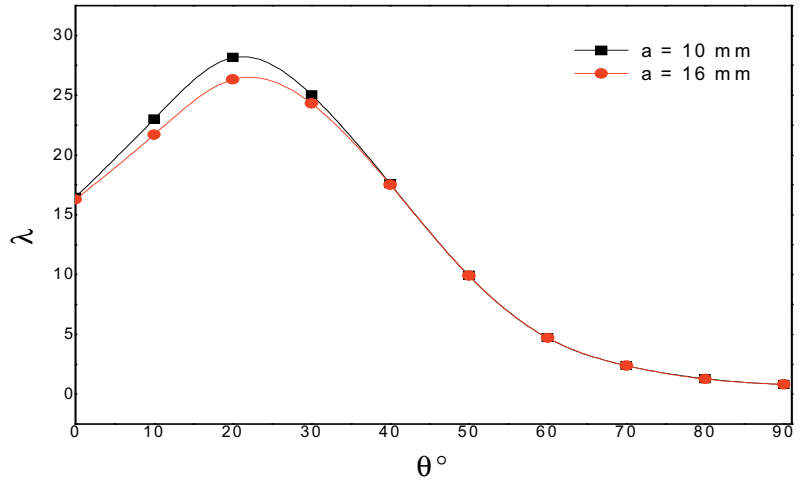

Fig. 11. Influence de la taille de l'entaille sur le facteur de flambement en fonction de $\theta^{\circ}$, pour un pipe à deux entailles. ( Entaille située au milieu, l'autre juste en face ).

La figure 11, désigne l'évolution du facteur de flambement pour le pipe avec deux entailles opposées, et situé au milieu de ce dernier. $\lambda=27.574$, donc presque la même valeur pour les trois cas précédent.
4.2.4 Cas de trois entailles situées du même coté du pipe

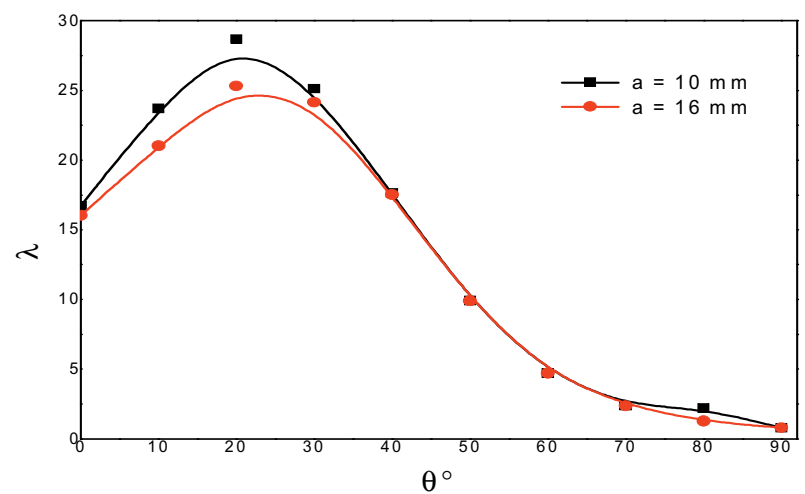

Fig. 12. Influence de la taille de l'entaille sur le facteur de flambement en fonction de $\theta^{\circ}$, pour un pipe à trois entailles.

pour le cas ou le pipeline a trois entailles, le paramètre $\lambda$ a presque la même valeur que précédemment. On conclu que l'emplacement de l'entaille, et même le nombre n'a pas d'effet significatif, on le vois bien dans les cas où la structure présente des défauts géométriques .

par contre si le pipe ne comporte pas d'entailles, le paramètre de flambement atteint des valeurs importantes $(\lambda \sim 65)$. dans ce cas l'a le pipe est moins fragile.

\section{5 conclusion}

L'étude présentée, a été faite pour déterminer l'effet des défauts géométriques dans un pipe stratifié soumis au flambement; l'ensemble des résultats obtenus montre que :

- Le coefficient de flambement atteint des valeurs maximales et importantes lorsque les fibres sont orientées généralement à $20^{\circ}$.

- Les valeurs minimales sont obtenues lorsque les fibres sont orientées dans l'intervalle de $70^{\circ}$ à $90^{\circ}$.

- la charge critique de flambement croît avec l'accroissement de l'épaisseur du pipe, ce qui montre l'augmentation de sa résistance.

- Le plus faible coefficient du flambement est obtenu pour le pipe avec les plus grandes tailles d'entailles et cela quelque soit la position et la taille de cette dernière.

- les valeurs maximales du flambement, appartiennent aux pipes munis de défauts géométriques . 
Les pipes avec des diamètres inférieurs a 500mm présentent un risque de telle sorte que le flambement atteint des valeurs maximales.

\section{References}

1. Yves Perrot, (Influence des propriétés de la matrice sur le comportement mécanique de matériaux composites verre/polyester utilisés en construction navale de plaisance - Cas des résines polyester limitant les émissions de styrène). Thèse. Université de Bretagne Sud. 28 Novembre (2006)

2. A. Beakou, A. Mohamed, (Influence of variable on the optimum winding angle of cylindrical laminated composites), composite structures 53 287-293. (2001)

3. Bouhafs Mohamed, Sereir Zouaoui, Alaa M. Chateauneuf(Effet des incertitudes mécaniques et géométriques sur la conception des pipelines en composite) JNC 17 - Poitiers 2011

4. Dvorak GJ, Prochazka P, Srinivas MV. Design and fabrication of submerged cylindrical laminates-I. Int J Solids Struct 36: 3917-43(1999).

5. Srinivas MV, Dvorak GJ, Prochazka P. Design and fabrication of submerged cylindrical laminates-II. Effect of fiber pre-stress. Int J Solids Struct 36:394576(1999). 